\title{
Costa Rica sin Fronteras
}

Pensar en Movimiento:

Revista de Ciencias del Ejercicio y la Salud

ISSN 1659-4436

Vol. 13, No.2, pp. 1-3

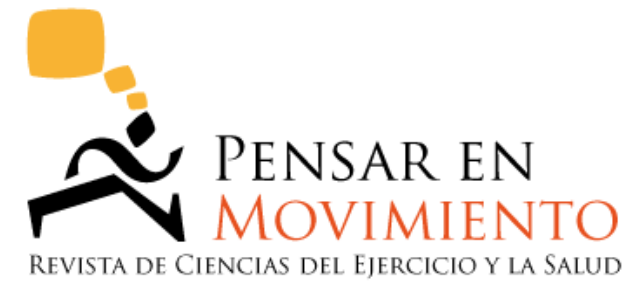

\section{COSTA RICA SIN FRONTERAS \#12}

En esta sección se publican los resúmenes en español de artículos que han sido publicados por investigadoras/es de universidades costarricenses en otras revistas en el mundo, con su debida referencia al trabajo original, y con una breve explicación de dónde se realizó la investigación. Los artículos originales han sido publicados en otros idiomas; las revistas tienen consejo editorial y manejan un proceso de revisión por pares.

Los resúmenes corresponden a estudios relacionados con las ciencias del ejercicio y la salud, que se conforman a los criterios generales de la revista, esto es, se trata de “... estudios experimentales o que hagan recomendaciones concretas para solucionar problemas o preguntas relevantes (...) trabajos originales o de meta-análisis." Solicitamos a las personas que han publicado este tipo de trabajos en otros idiomas que no los hagan saber, para incluir sus resúmenes aquí. 


\section{VALIDEZ PREDICTIVA DEL INDICE DE ADIPOSIDAD CORPORAL EN UNIVERSITARIOS COSTARRICENSES}

Carpio-Rivera, E., Hernández-Elizondo, J., Salicetti-Fonseca, A., Solera-Herrera, A. \& MoncadaJiménez, J. (2015). Predictive Validity of the Body Adiposity Index in Costa Rican Students. American Journal of Human Biology. 00:00-000. doi: 10.1002/ajhb.22800. Recuperado de: http://onlinelibrary.wiley.com/doi/10.1002/ajhb.22800/abstract

El objetivo de este estudio fue verificar la validez del índice de adiposidad corporal (BAI por sus siglas en inglés) en una muestra de estudiantes universitarios costarricenses. Para ello se utilizó como "estándar de oro" la absorciometría de energía dual de rayos X (DXA) para determinar el porcentaje de grasa corporal (BF\% por sus siglas en inglés). Por medio de coeficientes de correlación de Pearson y pruebas T de Student para muestras pareadas se determinó si existía asociación y diferencias significativas entre BAI y DXA BF\%. Asimismo se determinó si existía concordancia entre BAI y DXA BF\% por medio de los coeficientes de correlación de concordancia de Lin y los análisis de gráficos de Bland-Altman. Los resultados principales demuestran que existe una correlación significativa entre BAI y DXA BF\% para mujeres $(r=0.74)$ y hombres $(r=0.53)(p<0.001)$ y diferencias significativas entre métodos tanto para mujeres (BAl $=29.3 \pm 4.1 \%$ vs. $D X A=36.5 \pm$ $7.8 \%)$ como para los hombres $(B A I=24.8 \pm 3.7 \%$ vs $D X A=21.9 \pm 8.6 \%)(p<0.001)$. Por otra parte se encontró una concordancia pobre para ambos géneros. Los gráficos Bland-Altman muestran que el BAI subestima y sobreestima el BF\% en relación con el "estándar de oro" en mujeres y hombres respectivamente. En conclusión, el BAI mostró una baja concordancia de BF\% al compararse con los valores obtenidos por el DXA, por lo que no se recomienda su uso para predecir BF\% en esta muestra de estudiantes centroamericanos.

Este estudio se realizó en la Universidad de Costa Rica y es parte de un proyecto de investigación registrado y aprobado por la Vicerrectoría de Investigación de este ente educativo. Colaboradora: Andrea Solera Herrera.

La revista American Journal of Human Biology reporta un factor de impacto (JCR) de 1.7. 


\section{Cambios rápidos del peso corporal después de una parada de manos: un análisis metrológico}

Acuña-Espinoza, Alejandro, \& Aragón-Vargas, Luis Fernando (2015). Rapid Changes of Body Weight after a Headstand: A Metrological Analysis. PLOS ONE 10(5): e0124764. doi:10.1371/journal.pone.0124764

Varias organizaciones deportivas responsables por la lucha grecorromana amateur han emitido nuevos reglamentos, con los cuales se persigue disuadir a los practicantes de recurrir a los métodos extremos de pérdida de peso. A pesar de ello, en la cultura de la lucha grecorromana todavía persisten distintos métodos para "dar el peso", entre las cuales está el pararse de cabeza inmediatamente antes de subirse a la báscula. Este procedimiento, según la creencia, sirve para disminuir la masa corporal registrada entre 250 y $500 \mathrm{~g}$ (un peso entre 2.45 y $4.89 \mathrm{~N}$ ). El objetivo de este estudio fue comparar las posibles diferencias entre el procedimiento de parada de cabeza (PC) y una medición normal (CON) del peso, utilizando un método de análisis metrológico definido por la Asociación Europea de Institutos Nacionales de Metrología. Se pesaron diecisiete hombres adultos sobre una plataforma de fuerza antes y después de pararse de cabeza o de mantenerse en posición de pie por 30s. El orden de los tratamientos se asignó aleatoriamente. El peso postest $(640.7 \pm 62.8 \mathrm{~N}$, media \pm de) fue significativamente mayor que el pretest $(640.3 \pm 62.7 \mathrm{~N}, \mathrm{p}<0.0001)$ para ambos tratamientos. No se encontró interacción entre tratamiento y tiempo ni se identificó una diferencia significativa entre el peso CON y el peso PC $(640.6 \pm 62.8 \mathrm{~N}$ y $640.9 \pm 62.9 \mathrm{~N}$, respectivamente, $p=0.3815)$. Las pruebas metrológicas sugieren que las diferencias estadísticas encontradas están asociadas a los errores de medición de la plataforma de fuerza en todos y cada uno de los intervalos. La diferencia de $45 \mathrm{~g}(0.44 \mathrm{~N})$ encontrada entre pretest y postest se ubica dentro de los valores de incertidumbre identificados para el equipo $( \pm 110 \mathrm{~g}$ o $1.08 \mathrm{~N})$. En conclusión, una parada de cabeza de 30 segundos no tiene efecto sobre el peso corporal registrado. Las pequeñas variaciones obtenidas se le atribuyen a errores de medición asociadas al equipo utilizado. Este experimento ofrece evidencia empírica sistemática para colaborar con la eliminación de esta práctica injustificada de la comunidad de la lucha grecorromana.

Este estudio se realizó en el Centro de Investigación en Ciencias del Movimiento Humano de la Universidad de Costa Rica. Colaborador: Luis Fernando Aragón Vargas.

La revista PLoS ONE reporta un factor de impacto (JCR) para el año 2014 de 3.234. 